\title{
Glucose Transport in Crabtree-positive and Crabtree-negative Yeasts
}

\author{
By HENDRIK VAN URK, ERIK POSTMA, W. ALEXANDER SCHEFFERS \\ AND JOHANNES P. VAN DIJKEN* \\ Department of Microbiology and Enzymology, Delft University of Technology, Julianalaan 67, \\ 2628 BC Delft, The Netherlands
}

(Received 3 March 1989; revised 23 May 1989; accepted 30 May 1989)

\begin{abstract}
The kinetic parameters of glucose transport in four Crabtree-positive and four Crabtreenegative yeasts were determined. The organisms were grown in aerobic glucose-limited chemostats at a dilution rate of $0 \cdot 1 \mathrm{~h}^{-1}$. The results show a clear correlation between the presence of high-affinity glucose transport systems and the absence of aerobic fermentation upon addition of excess glucose to steady-state cultures. The presence of these $\mathrm{H}^{+}$-symport systems could be established by determination of intracellular accumulation of 6-deoxy- $\left[{ }^{3} \mathrm{H}\right]$ glucose and alkalinization of buffered cell suspensions upon addition of glucose. In contrast, the yeasts that did show aerobic alcoholic fermentation during these glucose pulse experiments had low-affinity facilitated-diffusion carriers only. In the yeasts examined the capacity of the glucose transport carriers was higher than the actual glucose consumption rates during the glucose pulse experiments. The relationship between the rate of sugar consumption and the rate of alcoholic fermentation was studied in detail with Saccharomyces cerevisiae. When $S$. cerevisiae was pulsed with low amounts of glucose or mannose, in order to obtain submaximal sugar consumption rates, fermentation was already occurring at sugar consumption rates just above those which were maintained in the glucose-limited steady-state culture. The results are interpreted in relation with the Crabtree effect. In Crabtree-positive yeasts, an increase in the external glucose concentration may lead to unrestricted glucose uptake by facilitated diffusion and hence, to aerobic fermentation. In contrast, Crabtree-negative yeasts may restrict the entry of glucose by their regulated $\mathrm{H}^{+}$-symport systems and thus prevent the occurrence of overflow metabolism.
\end{abstract}

\section{INTRODUCTION}

For yeasts several glucose uptake systems have been described in the literature. Some yeasts can transport glucose by an inducible high-affinity $\left(K_{\mathrm{m}} 20-200 \mu \mathrm{M}\right)$ proton symport mechanism. Examples are: Kluyveromyces marxianus (Gasnier, 1987; Van den Broek et al., 1986) and Candida species (Spencer-Martins \& van Uden, 1985a; Postma et al., 1988). In a recent paper Loureiro-Dias (1988) reported, on the basis of a study with 205 different yeast species, that induction of this proton symport mechanism at low glucose concentrations is particularly common in the genera Rhodotorula, Candida and Hansenula. At high glucose concentrations this high-affinity transport is repressed and only a low-affinity carrier $\left(K_{\mathrm{m}} 2-3 \mathrm{~mm}\right)$ is detected (Gasnier, 1987; Spencer-Martins \& van Uden, 1985a). Proton symporters could not be detected in most of the Saccharomyces strains tested (Loureiro-Dias, 1988).

In Saccharomyces cerevisiae a facilitated-diffusion transport mechanism has been encountered (Romano, 1982). Also a phosphate-glucose group translocation mechanism has been suggested (Van Steveninck et al., 1985; Franzusoff \& Cirillo, 1982). Bisson \& Fraenkel (1983) and Lang \& Cirillo (1987) reported the presence of a so-called high-affinity transporter $\left(K_{\mathrm{m}} 1 \mathrm{~mm}\right)$. The occurrence of this system is dependent on the synthesis of hexokinases. It should be noted that this 'high-affinity' carrier has an affinity for glucose that is $\mathbf{1 0}$ to 50 times lower than the highaffinity carriers in, e.g., Kluyveromyces marxianus and Candida utilis (Gasnier, 1987; Postma et 
al., 1988). A low-affinity carrier $\left(K_{\mathrm{m}} 20-50 \mathrm{~mm}\right)$ is always present in $S$. cerevisiae; this carrier is not dependent on the presence of hexokinase activity (Lang \& Cirillo, 1987).

Yeasts exhibit characteristic responses to changes in the environmental sugar concentration. In organisms such as $S$. cerevisiae alcoholic fermentation is triggered when aerobic sugar-limited cultures are exposed to sugar excess. This response, which is instantaneous, is known as the short-term Crabtree effect (Petrik et al., 1983; van Urk et al., 1988) and is followed by long-term adaptation involving repression of respiratory enzymes (the long-term Crabtree effect). Crabtree-negative organisms such as Candida species do not exhibit this reponse (De Deken, 1966; Knöpfel, 1972; Petrik et al., 1983; Rieger et al., 1983).

In view of the differences in kinetics and mechanisms of glucose transport among yeasts it was of interest to study the possible relation between the occurrence of the short-term Crabtree effect and the mode of sugar transport. This paper presents a comparative study with eight different yeast species.

\section{METHODS}

Micro-organisms and growth conditions. Saccharomyces cerevisiae CBS 8066, Torulopsis glabrata CBS 138, Schizosaccharomyces pombe CBS 356, Brettanomyces intermedius CBS 1943, Candida utilis CBS 621, Hansenula nonfermentans CBS 5764, Kluyveromyces marxianus CBS 6556 and Pichia stipitis CBS 5773 were maintained on malt agar slopes. The organisms were grown aerobically in a glucose-limited chemostat at $D=0.1 \mathrm{~h}^{-1}$ on a mineral medium with ammonium sulphate as the nitrogen source at $\mathrm{pH} 5.0$ and $30^{\circ} \mathrm{C}$, as described by Bruinenberg $e t$ al. (1983), unless mentioned otherwise. The reservoir glucose concentration was $5 \mathrm{~g} \mathrm{l}^{-1}$.

Measurement of residual glucose concentrations. A quick sampling method, by which the culture fluid is transferred within $3 \mathrm{~s}$ into liquid nitrogen, was used as described by Postma et al. (1988).

Glucose and mannose transport assays. The assay described by Postma et al. (1988) was adopted. This method is based on the uptake of $\left[\mathrm{U}-{ }^{14} \mathrm{C}\right] \mathrm{glucose}$ or $\left[\mathrm{U}-{ }^{14} \mathrm{C}\right]$ mannose during a $5 \mathrm{~s}$ incubation period. Cells used in this assay were harvested from the chemostat and used without further treatment at a concentration of $2 \cdot 2-2 \cdot 6 \mathrm{~g} \mathrm{I}^{-1}$. For all species tested the uptake of sugar was linear with time for at least $20 \mathrm{~s}$. The specific activity of the label used was $0 \cdot 1-100 \mathrm{mCi} \mathrm{mmol}^{-1}\left(3.7 \mathrm{GBq} \mathrm{mmol}^{-1}\right)$. From the uptake measurements the kinetic parameters were calculated using Hanes plots (Postma et al., 1988).

Acccumulation of 6-deoxy- ${ }^{3} \mathrm{H}$ glucose. The assay was essentially the same as the glucose transport assay. A trace concentration of labelled 6-deoxyglucose $\left(70000 \mathrm{~d}\right.$.p.m.) was added to the cell suspension $\left(2.5 \mathrm{~g} \mathrm{l}^{-1}\right)$. It was established that in the organisms used equilibration of the intracellular and extracellular 6-deoxyglucose concentration was reached within $2 \mathrm{~min}$. The accumulation factor as presented in Table 3 was calculated assuming an intracellular volume of $2 \mathrm{ml}(\mathrm{g} \mathrm{dry} \mathrm{wt})^{-1}$. This assumption is based on values reported by Beaven et al. (1982) and Conway \& Downey (1950).

Measurement of glucose-dependent alkalinization. After harvesting from steady-state cultures by centrifugation, cells were washed twice in potassium phthalate buffer $(1.25 \mathrm{mM}, \mathrm{pH} 5.0$, room temperature) and resuspended in this buffer to a final concentration of $10 \mathrm{~g} \mathrm{dry} \mathrm{wt} 1^{-1}$. This suspension was aerated at room temperature until used for measurement (within $30 \mathrm{~min}$ ). The assay was done in a magnetically stirred vessel containing $5 \mathrm{ml}$ of suspension kept at $30^{\circ} \mathrm{C}$ unless stated otherwise. It was established, using a Clark-type oxygen electrode, that no oxygen limitation occurred during the experiment. The following configuration, with a reaction time of less than $1 \mathrm{~s}$, was used: an Ankersmit A 216 sensitive pH electrode was connected to a Philips PW $9421 \mathrm{pH}$ meter. The signal was recorded with a Kipp BD 40 recorder (paper feed velocity: $10 \mathrm{~cm} \mathrm{~s}^{-1}$ ). The $\mathrm{pH}$ meter was used in the $\mathrm{mV}$ mode. To calculate $\mathrm{H}^{+}$uptake rates from the recordings an equilibration curve in the buffer or cell suspension was made with a standard $\mathrm{NaOH}$ solution.

Glucose and mannose pulse experiments. Glucose pulse experiments were done with all strains by adding $50 \mathrm{mM}-$ glucose to aerobic glucose-limited cultures pregrown at $D=0.1 \mathrm{~h}^{-1}$. Additionally, pulse experiments on $S$. cerevisiae were done by adding lower glucose or mannose concentrations $(0 \cdot 3-50 \mathrm{mM})$. During the pulse experiments samples were taken every 2-5 min and centrifuged immediately (within $15 \mathrm{~s}$ ) (van Urk et al., 1988). The supernatants were used for the determination of the glucose or mannose concentration. The initial glucose or mannose consumption rates were calculated from the tangent on the curve produced by plotting glucose or mannose concentrations versus time.

The oxygen consumption rates were determined by analysis of the off-gas from the fermentors using a Servomex OA-184 oxygen analyser, and a Beckman $\mathrm{CO}_{2}$ analyser for correction as described by van Urk et al. (1988).

Measurement of glucose and mannose concentrations. The glucose and mannose concentrations were determined with a Boehringer glucose kit (enzymic hexokinase/glucose-6-phosphate dehydrogenase/UV method). For the determination of mannose, phosphomannose isomerase (Boehringer) was added $\left(3 \mathrm{U} \mathrm{ml}^{-1}\right)$ to the assay mixture.

Radiochemicals. [U-14 ${ }^{14}$ lglucose and $\left[\mathrm{U}-{ }^{14} \mathrm{C}\right]$ mannose $\left(270 \mathrm{mCi} \mathrm{mmol}^{-1}\right)$ were obtained from Amersham (England) and 6-deoxy-[ $\left[{ }^{3} \mathrm{H}\right] \mathrm{glucose}$ (of unknown specific activity) from New England Nuclear. 
Table 1. Rates of glucose consumption by Crabtree-positive and Crabtree-negative yeasts

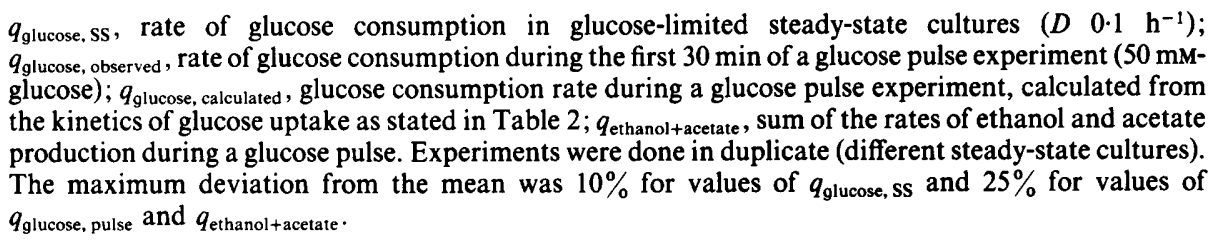

$q_{\text {glucose, ss }} q_{\text {glucose, observed }} q_{\text {glucose, calculated }} \quad q_{\text {ethanol+acetate }}$

Crabtree-positive yeasts

\begin{tabular}{|c|c|c|c|c|}
\hline S. cerevisiae & $1 \cdot 1$ & $5 \cdot 4$ & $18 \cdot 0$ & $6 \cdot 9$ \\
\hline$T$. glabrata & $1 \cdot 2$ & $7 \cdot 1$ & $98 \cdot 7$ & $9 \cdot 2$ \\
\hline Sch. pombe & $1 \cdot 3$ & $7 \cdot 1$ & 8.7 & 5 \\
\hline B. intermedius & $1 \cdot 0$ & $1 \cdot 1$ & $4 \cdot 5$ & 0.3 \\
\hline \multicolumn{5}{|c|}{ rabtree-negative yeasts } \\
\hline C. utilis & $1 \cdot 1$ & $5 \cdot 0$ & $13 \cdot 8$ & $<0$ \\
\hline H. nonfermentans & $1 \cdot 3$ & 2.5 & $8 \cdot 1$ & 0 \\
\hline K. marxianus & $1 \cdot 4$ & 3.5 & $4 \cdot 2$ & 0 \\
\hline P. stipitis & $1 \cdot 3$ & $3 \cdot 3$ & $6 \cdot 0$ & 0 \\
\hline
\end{tabular}

\section{RESULTS}

Glucose consumption rates during glucose pulse experiments

All four Crabtree-positive yeasts selected for our study exhibited an immediate alcoholic fermentation when excess glucose was added to steady-state cultures grown under glucose limitation. Brettanomyces intermedius behaved exceptionally: its fermentive response was rather weak (Table 1). This coincided with much lower rates of glucose consumption as compared to the other Crabtree-positive yeasts. Alcoholic fermentation after the transition to excess glucose was absent in the Crabtree-negative species. In this group $C$. utilis stood out by its high rate of sugar consumption. The results of these pulse experiments show that all the yeasts (except $B$. intermedius) responded to a glucose pulse by an increase in the rate of sugar consumption. This phenomenon does not require enzyme synthesis since the effect was instantaneous (within 5 min). Rather, it must result from a higher enzyme activity due to increased substrate concentrations for the various enzymes of glucose metabolism, including the uptake systems.

\section{Kinetic parameters of glucose uptake in cells grown under glucose limitation}

In order to ascertain to what extent the enhanced rates of glucose consumption after a glucose pulse are prescribed by the glucose uptake system, the kinetics of glucose uptake were determined for all yeast species listed in Table 1 . The uptake of $\left[{ }^{14} \mathrm{C}\right]$ glucose was determined in $5 \mathrm{~s}$ incubations. This short incubation time was chosen in order to minimize the influence of further metabolism on the transport assay (Postma et al., 1988). Except for Schizosaccharomyces pombe and Pichia stipitis, a Hanes plot yielded broken curves indicating the presence of multiple uptake systems. In the case of Hansenula nonfermentans a triphasic curve was obtained. The $K_{\mathrm{m}}$ values for glucose uptake among the various yeasts differed up to 1000 -fold. In the Crabtreepositive yeasts, except $B$. intermedius, affinity constants were in the millimolar range. In the Crabtree-negative yeasts and in $B$. intermedius affinity constants in the micromolar range were encountered (Table 2).

\section{Calculation of glucose consumption rates with the kinetic parameters from the uptake assays}

The kinetic parameters of the various uptake systems can be used to calculate the steady-state flux of glucose into the cells, provided that the residual substrate concentration in these steadystate cultures is known. This parameter was determined for all yeasts by the rapid sampling technique (see Methods). The residual substrate concentration was a reflection of the transport system present in a particular yeast. In cultures of yeasts possessing high-affinity carriers the 
Table 2. Apparent affinities $\left(K_{m}\right)$ and capacities $(V)$ of glucose uptake by cells growing under glucose limitation $\left(D 0.1 \mathrm{~h}^{-1}\right)$

Experiments were done with cells from two different steady-state cultures; the maximum deviation from the mean values was $20 \%$.
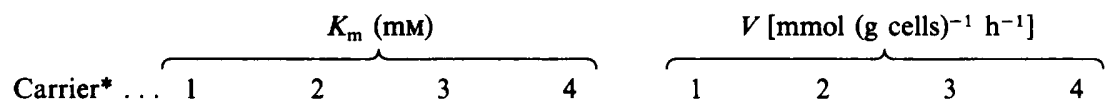

Crabtree-positive yeasts

S. cerevisiae

T. glabrata

Sch. pombe

B. intermedius

$\begin{array}{llll}- & - & 1.0 & 20.0\end{array}$

$8 \cdot 0$

$\begin{array}{cccc}- & - & 12.0 & 9 \cdot 0 \\ - & - & 31 \cdot 2 & 93.0 \\ - & - & 9 \cdot 0 & - \\ 2 \cdot 1 & 2.4 & - & -\end{array}$

Crabtree-negative yeasts

C. utilis

H. nonfermentans

K. marxianus

P. stipitis

$\begin{array}{cccc}- & - & 1.2 & 18 \cdot 0 \\ - & - & 1.5 & - \\ 0.030 & 0.6 & - & -\end{array}$

$-\quad 2 \cdot 1$

$\begin{array}{llllll}0.025 & 0.2 & - & - & 8.4 & 5.4\end{array}$

$\begin{array}{llllll}0.020 & 0.2 & 3.1 & - & 3.6 & 1.5\end{array}$

$1 \cdot 5$

1.2

$6 \cdot 0$

* In the text carriers 1 and 2 are denoted as high-affinity systems and 3 and 4 as low-affinity carriers.

- , Carrier not present.

Table 3. Residual glucose concentrations and cell yields in steady-state cultures growing under glucose limitation $\left(D 0.1 h^{-1}\right)$ and ratios of the calculated in situ glucose consumption rates to the observed rates

Experiments were done in duplicate (different steady-state cultures). The maximum deviation from the mean was $25 \%$ for values of residual glucose concentration and $5 \%$ for yield values.

\section{Residual glucose}

$(\mu \mathrm{M})$

Crabtree-positive yeasts

$S$. cerevisiae

T. glabrata

Sch. pombe

B. intermedius

Crabtree-negative yeasts

C. utilis

$H$. nonfermentans

K. marxianus

P. stipitis

\section{0}

100

160

20

5

15

35
Yield

[g (g glucose $)^{-1}$ ]

0.51

$0 \cdot 46$

0.44

0.52

0.51

0.51

0.48

0.45 $q_{\text {calculated }} / q_{\text {observed }}$

$1 \cdot 1$

$2 \cdot 4$

0.7

0.9

$1 \cdot 4$

$1 \cdot 5$

$0 \cdot 7$

$1 \cdot 2$

residual substrate concentration was lower than in those having low-affinity carriers only (compare Tables 2 and 3 ).

The in situ rate of glucose uptake in steady-state cultures can be calculated according to the Michaelis-Menten equation

$$
q_{\text {calculated }}=\sum_{n} V_{n} \frac{S}{K_{\mathrm{m}, n}+S} \quad(n=1,2,3,4)
$$

$V_{n} \quad$ = capacity of carrier no. $n$ (Table 2 )

$K_{\mathrm{m}, n}=$ affinity constant of carrier no. $n$ (Table 2)

$S=$ residual substrate concentration

This in situ rate, which is the sum of the contributions of the individual uptake systems, should be equal to the rate observed

$D \quad=$ dilution rate $\left(\mathrm{h}^{-1}\right)$

$$
q_{\text {observed }}=D / Y
$$

$Y=$ cell yield $\left[\mathrm{g}\right.$ cells $\left.(\mathrm{mmol} \text { glucose })^{-1}\right]$ 

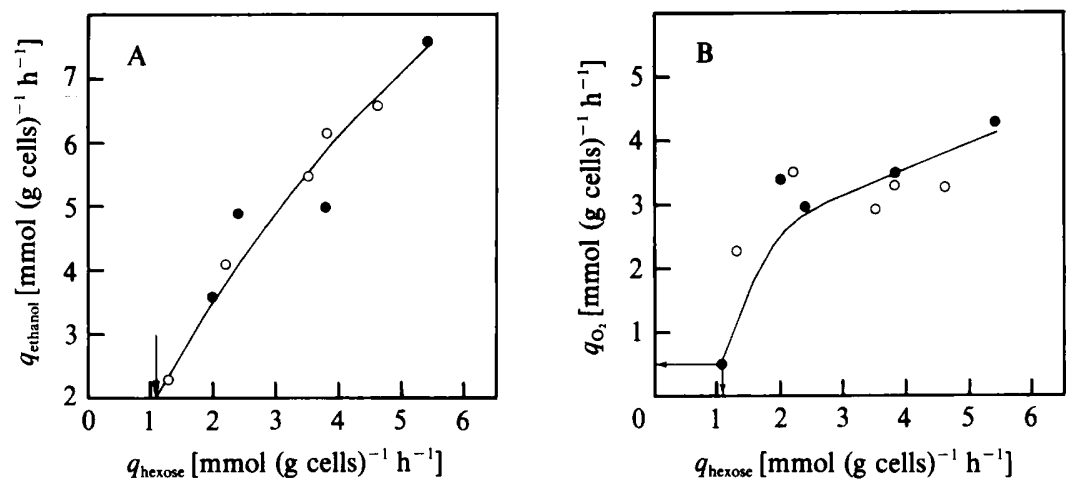

Fig. 1. A, Relationship between the initial rate of glucose $(O)$ or mannose $(O)$ consumption $\left(q_{\text {hexose }}\right)$ and the rate of ethanol production by $S$. cerevisiae upon addition of different amounts of hexose to glucose-

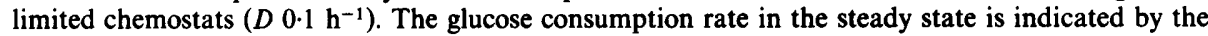
arrow. B, Specific rate of oxygen consumption as a function of the rate of sugar consumption during pulse experiments with different concentrations of glucose $(O)$ or mannose $(O)$. The arrows indicate the situation in the steady state.

Thus, the ratio $q_{\text {calculated }} / q_{\text {observed }}$ should be 1 . This requirement was approximately met for all yeasts tested, except Torulopsis glabrata (Table 3).

In a similar way the glucose consumption rates during glucose pulse experiments, in which the initial glucose concentration was $50 \mathrm{~mm}$, can be calculated and compared to the observed glucose consumption rates (Table 1). It is clear from this comparison that most of the yeasts have a higher capacity for glucose transport than the observed glucose consumption rates during a pulse (Table 1). Only $K$. marxianus and Sch. pombe showed about the same glucose consumption rates as those calculated from the transport parameters.

\section{Relation between the rates of glucose consumption and alcoholic fermentation in $S$. cerevisiae}

The instantaneous alcoholic fermentation in Crabtree-positive yeasts can be considered as overflow metabolism, triggered by an enhanced rate of sugar uptake. It was therefore of interest to study the relation between the sugar consumption rate during these pulse experiments and the fermentation rate. In order to establish this relation, sugar pulse experiments with different amounts of glucose or mannose $(0 \cdot 3-50 \mathrm{~mm})$ were done with glucose-limited cultures of $S$. cerevisiae. The rationale of choosing mannose for the experiments was based on the reports by Kotyk (1967) and Cirillo (1968) that $S$. cerevisiae has a lower affinity for this sugar than for glucose. However, it was established in our experiments that the overall affinity constants of $S$. cerevisiae for glucose and mannose under these conditions were almost equal, namely, 1.0 and $1.2 \mathrm{mM}$, respectively (results not shown). Moreover, mannose uptake measurements with [U- ${ }^{14} \mathrm{C}$ ]mannose revealed a $K_{\mathrm{m}}$ value for the uptake of this sugar of $1.8 \mathrm{~mm}$ (results not shown). Pulse experiments with a range of sugar concentrations revealed that $S$. cerevisiae had already started to ferment at sugar consumption rates which were just above the steady-state consumption rate (Fig. 1A). The oxygen consumption rate at a low sugar consumption rate (Fig. 1B) was about $4 \mathrm{mmol}$ (g cells) $)^{-1} \mathrm{~h}^{-1}$, against approximately $6.3 \mathrm{mmol}(\mathrm{g} \mathrm{cells})^{-1} \mathrm{~h}^{-1}$ at the highest glucose consumption rate measured. This indicates that at the low sugar consumption rates the oxygen consumption rates were not limiting.

\section{Mechanism of glucose uptake in Crabtree-positive and Crabtree-negative yeasts}

As outlined above, the Crabtree-negative yeasts and $B$. intermedius possess uptake systems for glucose that have much higher substrate affinities than those of the Crabtree-positive yeasts. The high-affinity uptake probably occurs via an energy-requiring process. Firstly, in yeasts possessing one or two high-affinity systems, alkalinization of suspensions was observed upon glucose addition (Fig. 2; Table 4). The proton/glucose stoichiometry was between 0.3 and 0.5 
A

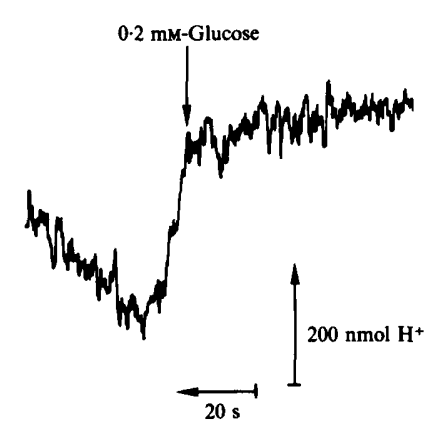

B

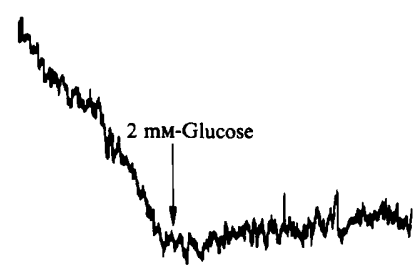

$200 \mathrm{nmol} \mathrm{H}^{+}$

Fig. 2. Typical recordings of $\mathrm{pH}$ in aerobic cell suspensions. Glucose was added at the time indicated. A, $H$. nonfermentans; B, $T$. glabrata.

Table 4. Accumulation of 6-deoxyglucose, maximum rate of glucose-induced alkalinization and $\mathrm{H}^{+} /$glucose uptake ratio in Crabtree-positive and Crabtree-negative yeasts

Cells were pre-grown under glucose limitation $\left(D 0.1 \mathrm{~h}^{-1}\right)$ except for $K$. marxianus (see Methods). Values for 6-deoxyglucose accumulation are from a single experiment on each yeast. Alkalinization experiments were done in duplicate with cells from two different steady-state cultures; the maximum deviation from the mean for the $V_{\mathrm{H}^{+}}$values was $20 \%$.

6-Deoxy-[$\left[{ }^{3} \mathrm{H}\right] g l u c o s e$
accumulation
$(-$ fold $)$

Crabtree-positive yeasts

\section{S. cerevisiae}

T. glabrata

Sch. pombe

B. intermedius

Crabtree-negative yeasts

C. utilis

$H$. nonfermentans

K. marxianus

P. stipitis

$$
\begin{gathered}
V_{\mathrm{H}^{+}} \text {of } \\
\text { glucose-dependent } \\
\text { alkalinization } \\
{\left[\mathrm{mmol}(\mathrm{g} \text { cells })^{-1} \mathrm{~h}^{-1}\right. \text { ] }}
\end{gathered}
$$

$$
\begin{aligned}
& \mathrm{H}^{+} / \text {glucose } \\
& \text { ratio }
\end{aligned}
$$

* Since the assays of glucose uptake and $\mathrm{H}^{+}$uptake were done with cells cultivated under different conditions, a ratio cannot be given in this case.

which is low compared to a theoretical minimal value of 1 . An explanation for this low value may be derived from Fig. 2A. Upon addition of glucose an alkalinization is observed, but, after about $10 \mathrm{~s}$, a rapid acidification of the buffer is seen. This acidification is probably due to excretion of $\mathrm{H}^{+}$by the glucose-stimulated plasma membrane ATPase, and to production of $\mathrm{CO}_{2}$ during the metabolism of glucose. Therefore, the alkalinization rates listed in Table 4 are the result of the $\mathrm{H}^{+}$/glucose symport and the acidification. With $K$. marxianus no measurable glucose-dependent alkalinization could be observed when the organism was pre-grown in chemostat culture under the standard conditions, which are sub-optimal for this organism $\left(30^{\circ} \mathrm{C}\right.$, $\left.D 0.1 \mathrm{~h}^{-1}\right)$. Instead, upon addition of glucose a rapid acidification was observed. The acidification rate under these conditions is probably higher than the alkalinization rate upon 
addition of glucose. However, when $K$. marxianus was grown at $40{ }^{\circ} \mathrm{C}$ and $D=0 \cdot 2 \mathrm{~h}^{-1}$ a pattern similar to that of the other Crabtree-negative yeasts was observed, which indicates that this yeast also possesses an $\mathrm{H}^{+}$/glucose symport system under glucose-limited conditions.

A second method to determine whether glucose is transported via an active uptake system was the measurement of 6-deoxyglucose accumulation. Only in yeasts possessing high-affinity uptake systems was 6-deoxyglucose accumulated against a concentration gradient. The accumulation was between 60 - and 200 -fold.

\section{DISCUSSION}

The results show that the eight yeast species studied can be divided into two groups with respect to the mechanism of glucose uptake. Those possessing high-affinity uptake systems accumulate glucose via an energy-requiring process, namely $\mathrm{H}^{+}$-symport (Tables 2 and 4). Like C. utilis (Postma et al., 1988), B. intermedius, $H$. nonfermentans and $P$. stipitis have a glucose uptake system with very high affinity $\left(K_{\mathrm{m}} 0.015-0.30 \mathrm{~mm}\right)$ also. This has not been reported previously for these yeasts. The other group, consisting of $S$. cerevisiae, $T$. glabrata and $S c h$. pombe, only exhibited low-affinity uptake systems. In these organisms glucose uptake probably proceeds by facilitated diffusion only, since sugar transport was not associated with alkalinization and 6-deoxyglucose was not accumulated (Tables 2 and 4). The residual substrate concentration in glucose-limited steady-state cultures of these three yeasts was significantly higher than in those of the other yeasts (Table 3).

Two groups of yeasts can also be identified on the basis of their physiological response after transition from limiting glucose to excess glucose, namely Crabtree-positive and Crabtreenegative yeasts. $B$. intermedius has an intermediate position between these two groups. This yeast is known as a Crabtree-positive organism since it shows significant production of ethanol and acetate after prolonged cultivation in strictly aerobic batch cultures (Wijsman et al., 1984). However, when pregrown under glucose limitation, it showed only a weak fermentative response to a glucose pulse and this coincided with the presence of a high-affinity uptake system as found in the Crabtree-negative yeasts.

The pulse experiments with $S$. cerevisiae (Fig. 1) reveal that overflow metabolism occurs even if the rate of glucose consumption is only slightly higher than that of the steady-state cultures. This is an interesting observation because in our strain, grown under glucose-limitation, glucose consumption rates up to $4.2 \mathrm{mmol}(\mathrm{g} \text { cells })^{-1} \mathrm{~h}^{-1}$ can be maintained without fermentation occurring (Postma et al., 1989). In contrast to $S$. cerevisiae, B. intermedius did not show an enhanced glucose consumption rate after addition of $50 \mathrm{~mm}$-glucose (Table 1). It can thus be argued that the presence of the different sugar uptake systems in yeasts (Tables 2 and 4) may play a role in the short-term Crabtree effect.

Although in a qualitative way the correlation between the modes of glucose uptake and the physiological responses of the eight yeasts appears clear-cut, a closer examination of the quantitative aspects of glucose consumption yields a more complicated picture. In all the yeasts examined the total capacity of the uptake systems exceeded the rate of glucose consumption after a pulse (Table 1). This, however, occurred to only a very limited extent in $S c h$. pombe and $K$. marxianus. In the other yeasts, both Crabtree-positive and Crabtree-negative species, glucose consumption rates were 2- to 15 -fold lower than the glucose uptake capacities of steady-state cells. Thus, at first sight, the occurrence of alcoholic fermentation in the Crabtree-positive organisms cannot be explained as a totally uncontrolled entry of glucose in the cell at a rate prescribed by the capacity of the uptake systems. Apparently, the flux through glycolysis in these organisms is determined by additional factors. Nevertheless, the way in which this modulation of uptake is accomplished in the two groups of yeasts is probably different. It is known that the high-affinity carriers of $C$. wickerhamii and $K$. marxianus are subject to a very rapid catabolite inactivation, occurring within a few minutes of glucose addition (Spencer-Martins \& van Uden, $1985 b$; De Bruijne et al., 1988). The rate of inactivation of the low-affinity carriers in Saccharomyces species is rather low and requires hours (Lagunas, 1986). Since the initial, rapid loss of activity during catabolite inactivation of enzymes is primarily caused by covalent 
modification via phosphorylation (Holzer, 1976), it may be argued that in the Crabtree-positive yeasts this type of modulation of the activity of the glucose carrier probably does not occur. In these organisms other mechanisms might be involved in the rapid feed-back regulation of carrier activity.

We thank Professor dr J. G. Kuenen for valuable discussions and Dr P. J. A. Van den Broek for kindly providing the labelled 6-deoxyglucose.

\section{REFERENCES}

Beaven, M. J., Charpentier, C. \& Rose, A. H. (1982). Production and tolerance of ethanol in relation to phospholipid fatty-acyl composition in Saccharomyces cerevisiae NCYC 431. Journal of General Microbiology 128, 1447-1455.

Bisson, L. F. \& FraENKEL, D. G. (1983). Involvement of kinases in glucose and fructose uptake by Saccharomyces cerevisiae. Proceedings of the National Academy of Sciences of the United States of America 80, $1730-1734$.

BRUinenberg, P. M., van Dijken, J. P. \& Scheffers, W. A. (1983). An enzymic analysis of NADPH production and consumption in Candida utilis. Journal of General Microbiology 129, 965-971.

CIRILLo, V. P. (1968). Relationship between sugar structure and competition for the sugar transport system in bakers' yeast. Journal of Bacteriology 95, 603-611.

Conway, E. J. \& Downey, M. (1950). An outer metabolic region of the yeast cell. Biochemical Journal 47, 347-355.

De Bruine, A. W., Schuddemat, J., Van den Broek, P. J. A. \& VAN StEVEnINCK, J. (1988). Regulation of sugar transport systems of Kluyveromyces marxianus: the role of carbohydrates and their catabolism. Biochimica et biophysica acta 939, 569-576.

De Deken, R. H. (1966). The Crabtree effect: a regulatory system in yeast. Journal of General Microbiology 44, 149-156.

Franzusoff, A. \& Cirillo, V. P. (1982). Uptake regulation and phosphorylation of 2-deoxy-D-glucose by wild-type and single-kinase strains of Saccharomyces cerevisiae. Biochimica et biophysica acta 688, 295-304.

GASNIER, B. (1987). Characterization of low- and highaffinity glucose transports in the yeast Kluyveromyces marxianus. Biochimica et biophysica acta 903, 425-433.

HoLzer, H. (1976). Catabolite inactivation in yeast. Trends in Biochemical Sciences 1, 178-181.

KNÖPFEL, H. P. (1972). Zum Crabtree-Effekt bei Saccharomyces cerevisiae und Candida tropicalis. Thesis no. 4906, ETH, Zurich.

KoTYK, A. (1967). Properties of the sugar carrier in bakers' yeast. II. Specificity of transport. Folia microbiclogica 12, 121-131.

LAGUNAS, R. (1986). Misconceptions about the energy metabolism of Saccharomyces cerevisiae. Yeast 2, 221-228.

LANG, J. M. \& CiRILLo, V. P. (1987). Glucose transport in a kinaseless Saccharomyces cerevisiae mutant. Journal of Bacteriology 169, 2932-2937.

LOUREIRO-DiAS, M. C. (1988). Movements of protons coupled to glucose transport in yeasts. A comparative study among 248 yeast strains. Antonie van Leeuwenhoek 54, 331-343.
Petrik, M., KäPPeli, O. \& Fiechter, A. (1983). An expanded concept for the glucose effect in the yeast Saccharomyces uvarum: involvement of short- and long-term regulation. Journal of General Microbiology 129, 43-49.

Postma, E., Scheffers, W. A. \& van Dijken, J. P. (1988). Adaptation of the kinetics of glucose transport to environmental conditions in the yeast Candida utilis CBS 621 : a continuous-culture study. Journal of General Microbiology 134, 1109-1116.

Postma, E., Verduyn, C., Scheffers, W. A. \& van DiJKEN, J. P. (1989). Enzymic analysis of the Crabtree effect in glucose-limited chemostat cultures of Saccharomyces cerevisiae. Applied and Environmental Microbiology 55, 468-477.

Rieger, M., KäPPELI, O. \& Fiechter, A. (1983). The role of limited respiration in the incomplete oxidation of glucose by Saccharomyces cerevisiae. Journal of General Microbiology 129, 653-661.

Romano, A. H. (1982). Facilitated diffusion of 6deoxy-D-glucose in bakers' yeast: evidence against phosphorylation-associated transport of glucose. Journal of Bacteriology 152, 1295-1297.

SPENCER-MARTINS, I. \& VAN UDEN, N. (1985a). Catabolite interconversion of glucose transport systems in the yeast Candida wickerhamii. Biochimica et biophysica acta 812, 168-172.

SPENCER-MARTINS, I. \& VAN UDEN, N. (1985b). Inactivation of active glucose transport in Candida wickerhamii is triggered by exocellular glucose. FEMS Microbiology Letters 28, 277-279.

van URK, H., MaK, P. R., Scheffers, W. A. \& vaN DiJKEN, J. P. (1988). Metabolic responses of Saccharomyces cerevisiae CBS 8066 and Candida utilis CBS 621 upon transition from glucose limitation to glucose excess. Yeast 4, 283-291.

VAN DEN Broek, P. J. A., Schuddemat, J., VAN Leeuwen, C. C. M. \& Van Steveninck, J. (1986). Characterization of 2-deoxyglucose and 6-deoxyglucose transport in Kluyveromyces marxianus: evidence for two different transport mechanisms. Biochimica et biophysica acta 860, 626-631.

Van Steveninck, J., Tiussen, J. P. F., Dubbelman, T. M. A. R. \& VAN Den BRoek, P. J. A. (1985). The role of polyphosphates in sugar transport across the plasma membrane of yeast. In. Environmental Regulation of Microbial Metabolism (FEMS Symposium no. 23), pp. 377-384. Edited by I. S. Kulaev, E. A. Dawes \& D. W. Tempest. London \& New York: Academic Press.

Wijsman, M. R., van Dijken, J. P., van KleefF, B. H. A. \& Scheffers, W. A. (1984). Inhibition of fermentation and growth in batch cultures of the yeast Brettanomyces intermedius upon a shift from aerobic to anaerobic conditions (Custers effect). Antonie van Leeuwenhoek 50, 183-192. 